\title{
Sull'integrazione delle funzioni quasi-periodiche astratte (*).
}

\author{
Memoria di Luigi Amerio (a Milano) \\ A Eurico Bompiani in occasione del suo Giubileo scientifico.
}

Sunto. - Si estende il teorema di BuHR, sull'mtegrazione delle funzioni quasi-periodiche a valori in uno spazio euclideo, alle funzioni quasi-periodiche a valori in uno spazio di BA.ACH uniformemente convesso. Si mostra poi, con esempi, che l'ipotesi di compattezza posta da BochNer non può, nel caso generale, essere sostituita da un'ipotesi di limitateza.

1. Il classico teorema di BoHn sull' integrazione delle funzioni quasi-periodiche (q. p.) a valori in uno spazio enclideo, is stato esteso dal BochNeR ( $\left.{ }^{1}\right)$ alle funzioni q. p. astratte, a valori in uno spazio $B$, di BANACH, qualsiasi. l'recisamente, data una siffatta funzione:

$$
x=f(t) \quad(t \in J=(-\infty \cdots+\infty)),
$$

il Bochner ha dimostrato che se lintegrale

$$
F(t)=\int_{0}^{t} f(\eta) d \eta
$$

ha traiettoria relativamente compatta, allora esso risulta q.p..

Tale enunciato estende quello di BOHR, sostituendo l'ipotesi di limitatezza dell'integrale. con l'altra assai più restrittiva (anche se consueta nelle teorie astratte) di compattezza.

Si presenta percio la questione (segnalata dallo stesso BochNer) di rico. noscere se, almeno in taluni spazi, l'ipotesi di compattezza possa attenuarsi, in modo da estendere inalterato l'enunciato di BoHR. In un recente lavoro $\left(^{2}\right)$

(*) Istituto Matematico del Politecnico di Milano.

(1) S. Bochner, Abstrakte fastperiodische Funktionen, "Acta math.», 61 (1933), p 149-184.

${ }^{2}$ ) L. Amerio, Sull' integrazione delle funzioni quasi-periodiche a valori in uno spazio hilbertiano, * Rend. Acc. Naz. dei Lincei», 28 (1960). 
ho dimostrato che questo effettivamente avviene se $B$ è uno spazio hilbertiano.

E spontaneo allora cercare di generalizzare il teorema così ottenuto agli spazi uniformemente convessi (o di CLARKson): questi spazi, caratterizzati da una espressiva condizione geometrica, comprendono, come casi particolari, gli spazi hilbertiani e gli spazi $L^{p}$ (con $p>1$ ).

Tale estensione è data nella presente Nota, nella quale si dimostra che se $f(t)$ è funzione q.p. a valori in uno spazio $K$, di Clarkson, e se l'integrale $F^{\prime}(t)$ ha traiettoria limitata, allora la funzione $F(t)$ risulta q.p.. La dimostrazione non $\grave{e}$, peraltro, una estensione di quella ottenuta per lo spazio hilbertiano: essa consiste nel provare che la traiettoria di $F(t)$ è relativamente compatta, ciò che consente di dedurre la tesi dal teorema di Bochner.

Si riconosce successivamente che, nel caso generale, l'ipotesi di compat. tezza, posta dal BochNer, non può essere eliminata. Si costruiscono infatti due esempi di funzioni $q$. p. (a valori rispettivamonte nello spazio $l^{\infty}$ delle successioni limitate e nello spazio $C$ delle funzioni continue nell'intervallo $0^{1-1}$ 1) le quali hanno integrali limitati ma non $q . p$.

2. Dimostriamo due lemmi, che utilizzeremo in seguito.

Lamia I. - Sia $F(t)$ una funzione debolmente quasi-periodica (d.q.p.), a valori in uno spazio $B$, di Banach, qualsiasi, e risulti, per una successione reale $\left\{\boldsymbol{z}_{i}\right\}$,

$$
\lim _{k \rightarrow \infty} F\left(t+z_{k}\right)=F_{z}(t)
$$

in tutto $J$.

$\grave{E}$ allora

$$
\operatorname{Sup}_{t \in J}\|F(t)\|=\operatorname{Sup}_{t \in J}\left\|F_{z}(t)\right\|
$$

Questo lemma si deduce facilmente dal teorema II e da un lemma dimostrati in un recente lavoro $\left({ }^{3}\right)$.

Cominciamó col ricordare il significato delle condizioni poste: preso

(3) L. A Merio, Funzioni debolmente quasi-periodiche, "Rend. Sem. Mat. di Padova,, 30 (1960), p. 288.301 . 
comunque un funzionale $\alpha \in B^{*}$ (spazio duale di $B$ ), la funzione $\alpha(F(t))$ risulta, per ipotesi, q. p. secondo BoHr e si ha

$$
\lim _{t \rightarrow \infty} \alpha\left(F\left(t+z_{k}\right)\right)=\alpha\left(F_{z}(t)\right)
$$

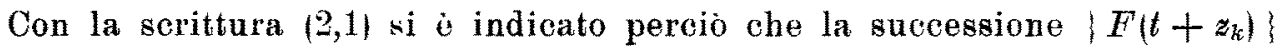
converge debolmente a $F_{z}(t)$, per ogni $t \in J$.

Per il lemma dianzi ricordato la convergenza è allora uniforme in $J$; $\alpha\left(F_{z}(t)\right)$ risulta pertanto q. p., ciò̀ $F_{z}(t)$ è d. q. p..

Inoltre, per l' uniformità della convergenza in $J$, si ha, dalla (2,3),

$$
\lim _{k \rightarrow-\infty} \alpha\left(F_{z}\left(t-z_{k}\right)\right)=\alpha(F(t))
$$

cioè

$$
\lim _{h \rightarrow \infty}^{*} F_{z}\left(t-z_{k}\right)=F(t)
$$

A causa della $(2,1)$, risulta poi

$$
\begin{aligned}
\left\|F_{z}(t)\right\| & \leq \min _{k \rightarrow \infty} \lim _{k \rightarrow \infty}\left\|F\left(t+z_{k}\right)\right\| \leq \\
& \leq \sup _{t \in t}\|F(t)\|,
\end{aligned}
$$

e quindi

$$
\operatorname{Sup}_{t \in J}\left\|F_{z}(t)\right\| \leq \operatorname{Sup}_{t \in J}\|F(t)\| .
$$

Analogamente, dalla $(2,+)$ si trae

$$
\operatorname{Sup}_{t \in J}\|F(t)\| \leq \operatorname{Sup}_{t \in J}\left\|F_{z}(t)\right\|
$$

\footnotetext{
e la $(2,2)$ risulta provata.
} 
Lemar II. - Se f(t) è funzione q.p. a valori in uno spazio B, di Banach, qualsiasi, e se l' integrale

$$
F(l)=\int_{0}^{l} f(\eta) d \eta
$$

è limitato, la funzione $F(t)$ risulta d.q.p..

Sia infatti $\alpha \in B^{*}$. Risulta

$$
\alpha(F(t))=\alpha\left(\int_{0}^{t} f(\eta) d \eta\right)=\int_{0}^{t} \alpha(f(\eta)) d \eta
$$

o quindi

$$
\left|\int_{0}^{1} \alpha(f(\eta)) d \eta\right| \leq\|\alpha\|\|F(t)\| \leq\|\alpha\| \operatorname{Sup}_{t \in J}\|F(t)\|<+\infty
$$

Poichè $\alpha(f(t))$ ò funzione q. p., ne segue la quasi-periodicita di $\alpha(F(t))$. Dall'arbitrarietà di $\alpha$ si deduce allora la tesi.

3. Ricordiamo che uno spazio $K \dot{\mathrm{e}}$ detto uniformemente convesso, $o d i$ Clarkson $\left({ }^{4}\right)$, quando in corrispondenza di ogni $\sigma$, con $0<\sigma \leq 2$, esistr un $\partial(0)>0$, tale che, per

$$
\|x\| \leq 1, \quad\|y\| \leq 1
$$

la condizione

$$
\|x-y\| \geq \sigma
$$

implichi

$(3,3)$

$$
\left\|\frac{x+y}{2}\right\| \leq 1-\delta(\sigma)
$$

(4) Cfr. MAHLon M. Dax, Normed linear spaces, "Springer, Berlin"; 1958, p. 112 
La funzione $\delta(\sigma)$ e detta il modulo di rotonditò di $K$. Gli spazi di Clarkson sono riflessivi ( $\left(^{5}\right)$, e quindi debolmente sequenzialmente completi $\left({ }^{6}\right)$.

Se supponiamo $x, y$ qualsiasi (e non necessariamente appartenenti alla sfera unitaria, come è richiesto dalle $(3,1)$, dalle $(3,2)$ e $(3,3)$ segue che $l a$ condizione

$$
\|x-y\| \geq \sigma \max (\|x\|,\|y\|)
$$

implica

$$
\left\|\frac{x+y}{2}\right\| \leq(1-\delta(\sigma)) \max (\|x\|,\|y\|)
$$

Cio premesso, dimostriamo il teorema seguente.

Se $f(t) \dot{e}$ funzione q.p., a valori in uno spazio $K$, uniformemente convesso, e se l'integrale

$$
F(t)=\int_{i}^{t} f(\eta) d \eta
$$

è limitato, allora la funzione $F(\ell)$ risulta q.p..

Basta provare, per il gia ricordato teorema di Bochner, che $F(t)$ ha traiettoria relativamente compatta.

In caso contrario, esistono un numero $\sigma>0$ ed una successione $\left\{z_{k}\right\}$ tali ehe risulti

$$
\left\|F\left(z_{j}\right)-F\left(z_{k}\right)\right\| \geq 2 \sigma \quad(j \neq k) .
$$

Possiamo supporre (estraendo eventualmente da $\left\{z_{k}\right\}$ una sottosuccessione, che indicheremo ancora con $\left\{z_{k} \mid\right\}$ che sia, uniformemente in $r$.

$$
\lim _{k \rightarrow \infty} f\left(t+z_{k}\right)=f_{z}(t)
$$

(5) Ofr. E. HuL, Funktional analysis and semigroups, "Am. Math. Soen, 1948, p. 22. (9) Cr. E. Hille, loc. eit. in (5) p. 24. 
e inoltre (a causa della limitatezza di $F(t)$ e della riflessività di $K$ )

$$
\lim _{k \rightarrow \infty}^{*} F\left(z_{k}\right)=c
$$

essendo $c$ un elemento di $K$.

Si ha poi, per ogni $t \in J$,

$$
\begin{aligned}
F\left(t+z_{k}\right) & =F\left(z_{k}\right)+\int_{z_{k}}^{z_{k}+t} f(\eta) d \eta= \\
& =F\left(z_{k}\right)+\int_{n}^{t} f\left(\eta+z_{k}\right) d \eta
\end{aligned}
$$

e quindi, per le $(3,7)$ e $(3,8)$, uniformemente in ogni intervallo limitato,

$$
\lim _{k \rightarrow \infty}^{*} F\left(t+z_{k}\right)=c+\int_{0}^{t} f_{z}(\eta) d \eta=F_{z}(t) .
$$

Osserviamo inoltro che $F(t)$ e funzione d.q.p., per il lemma II.

Risulta perció, in virtì del lemma I,

$$
\operatorname{Sup}_{t \in J}\left\|F_{\approx}(t)\right\|=\operatorname{Sup}_{t \in J}\|F(t)\|=M .
$$

Ora si ha, per le $(3,7)$ e $(3,9)$, in ogni fissato $t$,

$$
\begin{aligned}
\left\|F^{\prime}\left(t+z_{j}\right)-F\left(t+z_{k}\right)\right\|=\left\|F\left(z_{j}\right)-F\left(z_{k}\right)+\int_{0}^{t}\left(f\left(\eta+z_{j}\right)-f\left(\eta+z_{k}\right)\right) d \eta\right\| \geq \\
\quad \geq\left\|F\left(z_{j}\right)-F\left(z_{k}\right)\right\|-\left\|\int_{0}^{t}\left(f\left(\eta+z_{j}\right)-f\left(\eta+z_{k}\right)\right) d \eta\right\| \geq \\
\quad \geq 2 \sigma-\left\|\int_{0}^{t}\left(f\left(\eta+z_{j}\right)-f\left(\eta+z_{k}\right)\right) d \eta\right\| \geq \\
\geq \sigma \geq \sigma M^{-1} \max \left\{\left\|F\left(t+z_{j}\right)\right\|,\left\|F\left(t+z_{k}\right)\right\|\right\}
\end{aligned}
$$

quando siano $j, k \geq k_{t}$. 
Dalle $(3,5)$ e $(3,12)$ segue, per $j, k \geq k_{t}$,

$$
\begin{aligned}
\left\|\frac{F\left(t+z_{j}\right)+F\left(t+z_{k}\right)}{2}\right\| & \leq\left(1-\delta\left(\sigma M^{-1}\right)\right) \max \left(\left\|F\left(t+z_{j}\right)\right\|,\left\|F\left(t+z_{k}\right)\right\|\right) \leq \\
& \leq\left(1-\delta\left(\sigma M^{-1}\right)\right) M
\end{aligned}
$$

e quindi, per la $(3,10)$,

$$
\begin{aligned}
\left\|F_{z}(t)\right\| & \leq \min \lim _{(j, k) \rightarrow \infty}\left\|\frac{F\left(t+z_{j}\right)+F\left(t+z_{k}\right)}{2}\right\| \leq \\
& \leq\left(1-\delta\left(\sigma M^{-1}\right)\right) M .
\end{aligned}
$$

Ne segue

$$
\operatorname{Sup}_{t \in J}\left\|F_{z}(t)\right\| \leq\left(1-\delta\left(\sigma M^{-1}\right)\right) \operatorname{Sup}_{t \in J}\|F(t)\|<\operatorname{Sup}_{t \in J}\|F(t)\|,
$$

ciò che è assurdo, per la $(3,11)$.

4. Diamo degli esempi, per dimostrare che l'ipotesi di compattezza, posta dal Boohner, non può eliminarsi nel caso generale.

a) Sia $l^{\infty}$ lo spazio delle successioni numeriche limitate: se $x \in l^{\infty}$ sarà perciò

$$
\begin{gathered}
x=\left\{\xi_{1}, \ldots, \xi_{n}, \ldots\right\} \\
\|x\|=\operatorname{Sup}_{n}\left|\xi_{n}\right| .
\end{gathered}
$$

Consideriamo la funzione, a valori in $l^{\infty}$,

$$
f(t)=\left\{\lambda_{n} \cos \lambda_{n} t\right\}
$$

ove le costant1 $\lambda_{n}$ soddisfano alle condizioni

$$
\lambda_{n}>0, \quad \lim _{n \rightarrow \infty} \lambda_{n}=0
$$

Dimostriamo che $f(t) \grave{e}$ funzione q.p.. 
Preso infatti $\varepsilon>0$, si determini $n_{\varepsilon}$ in modo che, per $n>n_{\varepsilon}$, risulti

$$
\lambda_{n} \leq \frac{\varepsilon}{2}
$$

e quindi, qualunque sia $\tau$,

$$
\operatorname{Sup}_{t \in J}\left|\lambda_{n} \cos \lambda_{n}(t+\tau)-\lambda_{n} \cos \lambda_{n} t\right| \leq \varepsilon .
$$

Le funzioni (in numero finito)

$$
\lambda_{n} \text { cost } \lambda_{n} t \quad\left(n=1, \ldots, n_{\varepsilon}\right)
$$

sono egualmente q. p., cioè ammettono un comune insieme $E_{\bar{\varepsilon}}$, relativamente denso, di quasi-periodi $\tau$, relativi al valore $\varepsilon$.

Per $\tau \in E_{\mathrm{\varepsilon}}$ risulta allora

$$
\operatorname{Sup}_{t \in J}\left|\lambda_{n} \cos \lambda_{n}(t+\tau)-\lambda_{n} \cos \lambda_{n} t\right| \leq \varepsilon \quad\left(n=1, \ldots, n_{\varepsilon}\right)
$$

Dalle $(4,3)$ e $(4,4)$ segue, per $\tau \in E_{\varepsilon}$,

$$
\operatorname{Sup}_{t \in J}\|f(t+\tau)-f(t)\| \leq \varepsilon,
$$

cioè $f(t)$ risulta q. p..

Si ha poi

$$
F(t)=\int_{0}^{t} f(\eta) d \eta=\left\{\sin \lambda_{,} t\right\}
$$

e quindi

$$
\|F(t)\| \leq 1
$$

ciò̀ $F(t)$ è funzione limitata.

La funzione $F(t)$ non è, però, q. p..

Infatti i funzionali lineari

$$
\alpha_{n}(x)=\xi_{n} \quad(n=1,2, \ldots)
$$

hanno norma 1 :

$$
\left\|\alpha_{n}\right\|=1
$$


Si ha perciò

$$
\left|\alpha_{n}(F(t+\tau))-\alpha_{n}(F(t))\right| \leq\|F(t+\tau)-F(t)\|
$$

e, se $F(t)$ fosse q. p., le funzioni

$$
\alpha_{n}(F(t))=\sin \lambda_{n} t
$$

dovrebbero risultare egualmente quasi-periodiche. cioè ammettere, in corrispondenza di ogni $\varepsilon>0$, un comune insieme, $E_{\varepsilon}^{\prime}$, relativamente denso, di quasi-periodi $\tau$ : dovrebbe pertanto risultare

$$
\operatorname{Sup}_{t \in J}\left|\sin \lambda_{n}(t+\tau)-\sin \lambda_{n} t\right| \leq \varepsilon \quad\left(n \geq 1, \tau \in E_{\varepsilon}^{\prime}\right) .
$$

Ora questo non avviene, se è $\varepsilon<2$. Infatti si ha

$$
\begin{aligned}
& \operatorname{Sup}_{t \in J}|\sin (t+\tau)-\sin t|=\operatorname{Sup}_{t \in J}\left|\sin \left(t+\frac{\tau}{2}\right)-\sin \left(t-\frac{\tau}{2}\right)\right|= \\
& =2\left|\sin \frac{\tau}{2}\right|,
\end{aligned}
$$

e la condizione

$$
\operatorname{Sup}_{t \in J}|\sin (t+\tau)-\sin t| \leq \varepsilon
$$

equivale ad imporre a $\frac{\tau}{2}$ di appartenere agli intervalli

$$
v \pi-\frac{\delta}{2}^{1-1} v \pi+\frac{\delta_{\mathrm{e}}}{2} \quad(v=0, \pm 1, \pm 2, \ldots),
$$

ove $\delta_{\mathrm{s}}$ ̀े definito dalle condizioni

$$
\sin \frac{\delta_{\varepsilon}}{2}=\frac{\varepsilon}{2}, \quad 0<\delta_{\varepsilon}<\pi
$$

Pertanto l'insieme dei quasi-periodi della funzione sin $t$, relativi al valo. re $\varepsilon$, è costituito dagli intervalli

$$
2 \nu \pi-\delta_{\mathrm{q}}^{1-1} 2 \nu \pi+\delta_{\varepsilon} \quad(\nu=0, \pm 1, \pm 2, \ldots)
$$


Se consideriamo la funzione $\sin \lambda, t$ otteniamo, in corrispondenza, gli intervalli

$$
\frac{2 v \pi-\delta_{\varepsilon}^{1-1}}{\lambda_{n}} \cdot \frac{2 v \pi+\delta_{\varepsilon}}{\lambda_{n}} \quad(v=0, \pm 1, \pm 2, \ldots)
$$

La distanza tra due, consecutivi, di tali intervalli vale

$$
\frac{2(\nu+1) \pi-\delta_{\varepsilon}}{\lambda_{n}}-\frac{2 v \pi+\delta_{\varepsilon}}{\lambda_{n}}=\frac{2\left(\pi-\delta_{\varepsilon}\right)}{\lambda_{n}}=b_{n, \varepsilon}
$$

e misura l'ampiezza di inclusione relativa al valore $\varepsilon$.

Poichè risulta

$$
\lim _{n \rightarrow \infty} b_{n, \varepsilon}=+\infty
$$

si conchiude che le funzioni $\sin \lambda_{1} t$ non ammettono una ampiezza di inclusione comune, ciò̀ non sono egualmente q. p..

b) Sia $C$ lo spazio delle funzioni $x=x(\lambda)$, continue nell' intervallo $\Lambda=0^{1-1} 1$, con la norma

$$
\|x\|=\max _{\lambda \in \Lambda}|x(\lambda)|
$$

Indicata con $\left\{\lambda_{n}\right\}$ una successione soddisfacente alle condizioni

$$
1=\lambda_{1}>\lambda_{2}>\ldots>\lambda_{n}>\ldots, \quad \lim _{n \rightarrow \infty} \lambda_{n}=0,
$$

definiamo nella striscia $J \times \Lambda$ la funzione $x(t, \lambda)$ ponendo

$$
\left\{\begin{array}{l}
x(t, 0)=0 \\
x\left(t, \lambda_{n}\right)=\lambda_{n} \cos \lambda_{n} t \\
x(t, \lambda)=x\left(t, \lambda_{n}\right) \frac{\lambda-\lambda_{n+1}}{\lambda_{n}-\lambda_{n+1}}+x\left(t, \lambda_{n+1}\right) \frac{\lambda_{n}-\lambda}{\lambda_{n}-\lambda_{n+1}}, \text { per } \lambda_{n+1} \leq \lambda \leq \lambda_{n} .
\end{array}\right.
$$

E chiaro che, $x(t, \lambda)$ si può interpretare come una funzione $f(t)$, a valori in $C$ :

$$
f(t)=\{x(t, \lambda)\}
$$


Che $f(t)$ sia q. p., segue dalle $(4,5)$ e da quanto dimostrato in $a)$.

Infatti sia $\tau$ un quasi-periodo comune alle funzioni $x\left(t, \lambda_{n}\right)$ : l'insieme $E_{\varepsilon}$ dei numeri $\tau$, come si è visto in $a$ ), risulta relativamente denso.

Si ha, per $\tau \in E_{\varepsilon}$, e qualunque sia $n$,

$$
\operatorname{Sup}_{t \in J}\left|x\left(t+\tau, \lambda_{n}\right)-x\left(t, \lambda_{n}\right)\right| \leq \varepsilon
$$

e quindi, per $\lambda_{n+1} \leq \lambda \leq \lambda_{n}$,

$$
\operatorname{Sup}_{t \in J}|x(t+\tau, \lambda)-x(t, \lambda)| \leq \varepsilon\left(\frac{\lambda-\lambda_{n+1}}{\lambda_{n}-\lambda_{n+1}}+\frac{\lambda_{n}-\lambda}{\lambda_{n}-\lambda_{n+1}}\right)=\varepsilon .
$$

Perciò, qualunque sia $\lambda \in \Lambda$, risulta

$$
\operatorname{Sup}_{t \in J}|x(t+\tau, \lambda)-x(t, \lambda)| \leq \varepsilon
$$

Ne segue

$$
\begin{gathered}
\operatorname{Sup}_{t \in J}\|f(t+\tau)-f(t)\|=\operatorname{Sup}_{t \in J}\left\{\max _{\lambda \in \Lambda}|x(t+\tau, \lambda)-x(t, \lambda)|\right\}= \\
=\operatorname{Sup}_{\lambda \in \Lambda} \operatorname{Sup}_{t \in J}|x(t+\tau, \lambda)-x(t, \lambda)| \leq \varepsilon,
\end{gathered}
$$

cioè $f(t)$ è funzione q. p. (essendo anche continua).

Si ha poi

$$
F(t)=\int_{0}^{t} f(\eta) d \eta=\{\mathrm{X}(t, \lambda)\}
$$

avendo posto

$$
\mathrm{X}(t, \lambda)=\int_{0}^{t} x(\eta, \lambda) d \eta
$$

Ne segue

$$
\begin{aligned}
& \mathrm{X}(t, 0)=0 \\
& \mathrm{X}\left(t, \lambda_{n}\right)=\sin \lambda_{n} t^{i} \\
& \mathrm{X}(t, \lambda)=\mathrm{X}\left(t, \lambda_{n}\right) \frac{\lambda-\lambda_{n+1}}{\lambda_{n}-\lambda_{n+1}}+\mathrm{X}\left(t, \lambda_{n+1}\right) \frac{\lambda_{n}-\lambda}{\lambda_{n}-\lambda_{n+1}}, \text { per } \lambda_{n+1} \leq \lambda \leq \lambda_{n},
\end{aligned}
$$


e quindi

$$
\|F(t)\| \leq 1
$$

ciò̀ $F(t)$ è funzione limitata.

Però $F(t)$ non è q. p..

Si considerino infatti $i$ funzionali lineari

$$
\alpha_{n}(x(\lambda))=x\left(\lambda_{n}\right)
$$

Risulta

$$
\left\|\alpha_{n}\right\|=1
$$

e inoltre

$$
\alpha_{n}(F(t))=\mathbf{X}\left(t, \lambda_{n}\right)=\sin \lambda_{n} t
$$

Le funzioni $\sin \lambda_{n} t$, se $F(t)$ \&े q. p., debbono essere allora egualmente q. p.; circostanza che non si verifica per quanto dimostrato in $a$ ).

Percid la funzione $F(t)$ non è q. p.. 